\title{
Overexpression of the GmDREB2 gene increases proline accumulation and tolerance to drought stress in soybean plants
}

\author{
Thi Thanh Nhan Pham ${ }^{1}$, Huu Quan Nguyen ${ }^{1}$, Thi Ngoc Lan Nguyen ${ }^{1}$, Xuan Tan Dao ${ }^{1}$, Danh Thuong Sy ${ }^{1}$, Van \\ Son Le ${ }^{2}$, Hoang Mau Chu ${ }^{1, *}$
}

${ }^{1}$ Thainguyen University of Education, Thai Nguyen University, Thai Nguyen 250000, Vietnam

${ }^{2}$ Institute of Biotechnology, Vietnam Academy of Science and Technology, Hanoi 100000, Vietnam

\section{*Corresponding author: chuhoangmau@tnu.edu.vn}

\begin{abstract}
The dehydration responsive element binding (DREB) is a plant protein subfamily expressed when soybean plants face abiotic stresses. These DREB proteins are also considered to activate the transcription of drought-resistant genes. In this study, we present the determined results of relationships between overexpression of Glycine max DREB2 (GMDREB2) with the transcription level of Glycine max pyrroline-5-carboxylate synthetase (GMP5CS) gene, proline accumulation and drought tolerant ability transgenic soybean plants as the basis for selection of transgenic lines with high drought tolerance. GmDREB2 was inserted into a plant transgenic vector and the 35S-GmDREB2-cmyc construct was transferred into the soybean plants by Agrobacterium-mediated transformation. Recombinant GmDREB2 protein with a molecular weight of approximately $20 \mathrm{kDa}$ was expressed in four transgenic soybean lines in the $T_{1}$ generation. The GmP5CS gene was shown to have significantly $(P<0.05)$ increased expression in the $T_{2}$ transgenic soybean lines and higher than compared to non-transgenic plants with considering both in well watered condition and stressed conditions were from 1.06 to 1.31 -fold and 1.37 to 1.95 -fold, respectively. The proline content of four transgenic soybean lines increased $155.81 \%$ to $187.42 \%$ after five days in drought-stress conditions and increased from $180.52 \%$ to $233.74 \%$ after nine days under drought-stress conditions $(P<0.05)$. Therefore, the overexpression of GmDREB2 resulted in increasing transcription level of P5CS gene, proline accumulation and drought-stress tolerance of the transgenic soybean plants. The GmDREB2 transformation into soybean plants was confirmed by the results of genetically modified lines in the $T_{2}$ generation (T2-1, T2-6, T2-7, and T2-8) with higher drought tolerant ability than those of non-transgenic plants.
\end{abstract}

Keywords: Drought tolerance, GmDREB2 gene,proline content,transcription factors, transgenic soybean.

Abbreviations: GmDREB2_ Glycine max dehydration responsive element binding protein 2; $T_{0}, T_{1}, T_{2}$ generations of transgenic plants; WT_the wild-type plants (non-transgenic plants).

\section{Introduction}

Abiotic stress is the main cause leading to crop failures worldwide, causing damage to the average yield by more than $50 \%$ in key crops (Bray, 2004) which includes soybean plants. The soybean (Glycine max (L.) Merrill) is considered one of the most drought-sensitive crops and belong to poor drought tolerant cultivar. An approach to improve drought tolerance in soybean plants is the use of gene overexpression, which confers drought resistance. Characterization of soybean drought-related activities is due to the contribution of multiple genes, such as genes involved in proline biosynthesis (Chu et al., 2010), root-elongated genes (Lo et al., 2014). The question is that necessary to select a specific gene to increase drought tolerance of soybean plants. Agrobacterium-mediated transformation with functional genes to enhance abiotic stress tolerance of soybean plants have been published (Lo et al., 2015; Zhang et al.,2015). However, the problem is to select a specific gene with strong expression to enhance drought tolerance of soybean plants. A variety of genes in plants induced by abiotic-stress are activated by transcription factors, such as genes encoding DREB transcription factors. Therefore, enhancing gene expression encoding the transcription factor DREB is a promising approach for developing transgenic crops with high abiotic stress resistance and according to Hussain et al. (2011), this method seems to be more effective than the transformation of specific functional genes.

The Glycine max dehydration responsive element binding protein 2 (GmDREB2) belongs to DREB subfamily in soybean plants, which is responds to abiotic stresses, functionin activating the transcription of drought-resistant genes. The GMDREB2 gene functions as an important transcription factor activating genes involved in abiotic stress tolerance. Thus, using the GMDREB2 as a transgene was proven to be useful for improving the drought tolerant ability of transgenic plants (Chen et al., 2007; Mizoi et al., 2012; Chen et al., 2007; Dao et al., 2014). The AP2 domain of GmDREB2 consists of 60 amino acids, in which 11 amino acids are DNA 
biding site linked to the dehydration response factor (DRE) or GCC box (Tang et al., 2007; Dao et al., 2014). According to Li et al. (2005), cis-acting elements and trans factors play important roles in gene expression in response to droughtstress (Li et al., 2005). And overexpression of the GmDREB2 transcription factor increases the transcriptional levels of genes involved in the mechanism to cope with the droughtstresses (Chen et al., 2007; Kobayashi et al., 2008; Mizoi et al., 2012).

Many approaches for enhancing abiotic stress tolerance in plants have been interested in research by authors. Those are studies on gene expression in roots under drought (Janiak et al., 2016), contemporaneous overexpression of some genes in transgenic rice plants enhanced physiological tolerance and agronomic performance under adversely abiotic stress conditions (James et al., 2018). Accordingly, the overexpression of genes encoding transcription factors or genes involved in the accumulation of osmotic adjusting substances improved drought stress tolerance of crop plants (Soleimani et al., 2015; Kudo et al., 2017; Guan et al., 2018). Consequently, these are the important bases in research methods for improving the drought tolerant ability of soybean plants. So, the objective of this study was to determine whether there were relationships between overexpression of GmDREB2gene with the transcription level of GMDREB2 gene, proline accumulation and abioticstress tolerance in the transgenic soybean plants.

\section{Results}

\section{Transferring the 35S-GmDREB2-cmyc construct into the} soybeans

The 35S-GmDREB2-cmyc construct (Fig. 1) was transferred into soybeans by Agrobacterium-mediated transformation via the cotyledonary node. Transformed samples were cultured, regenerated and selected; the results are presented in Figure 2. After transferring results 35sGMDREB2-cmyc construct into 530 cotyledons of soybean cultivar DT84 and 257 samples were obtained and generated multiple shoots. After two rounds of selection by antibiotics at both the shoot formation and shoot-prolonged stages, 94 elongated shoots were obtained; they survived and grew well on the rooted media. At this stage, there were 58 shoots rooted and 32 in vitro plantlets with normal trunks and leaves. Their roots were selected to plant in pots, and 17 plants survived and developed normally in the greenhouse, with a success rate of $3.21 \%$. The non-transgenic soybean cotyledons were cultured on the shoot regeneration medium without selective antibiotics were used as controls (wild-type: WT).

\section{Verification of the integration of the GMDREB2 transgene into the soybean genomes at the $T_{0}$ generation}

Total DNA from the leaves of 17 transgenic soybean plants at the $T_{0}$ generation and non-transgenic (WT) plants were extracted and purified. The PCR reaction with the primer pair GMDREB2-BamHI/ GMDREB2-cmyc-Sacl was used to amplify the GmDREB2 gene from the genomic DNA. The results of PCR and agarose gel electrophoresis analysis showed there were $0.52 \mathrm{~kb}$ electrophoretic bands related to GmDREB2 gene in 14 plants of 17 transgenic plants, labelled
T0-1, T0-3, T0-4, T0-5, T0-6, T0-7, T0-8, T0-10, T0-11, T0-12, T0-13, T0-14, T0-15, and T0-17 (Fig.3A). Meanwhile, theGmDREB2 transgene was not observed in three transgenic soybean plants (TO-2, TO-9, TO-16) and the nontransgenic plants. These results could suggest that the genome of the fourteen transgenic soybean plants at the $T_{0}$ generation contained the GMDREB2 transgene. However, it was necessary to further assess whether the GMDREB2 transgene was incorporated into the genome of the transgenic plants. Hence, Southern blot was used to demonstrate that the GMDREB2 transgene was incorporated into the genome of the transgenic soybean plants.

Among the fourteen transgenic soybean plants with positive PCR products, there were eight stunted plants that grew poorly and six transgenic plants that developed normally. In all, five $T_{0}$ transgenic lines (T0-1, T0-4, TO-6, T0-7, and TO-8) were generated and confirmed by Southern blot assays (Fig.3B). The transgenic efficiency was $6 / 530$ or $1.13 \%$. Thus, it can be concluded that the GMDREB2 gene had been incorporated into the genome of the DT84 soybean cultivar.

\section{Expression analysis of the recombinant GMDREB2 protein in the transgenic soybean lines}

In these experiments, the five transgenic soybean lines in the $T_{1}$ generation (T1-1, T1-4, T1-6, T1-7, and T1-8) were used to analyse expression of the recombinant GmDREB2 protein. Total protein extracted from the leaves of five $T_{1}$ transgenic soybean lines was used for Western blotting with the c-myc antibody, and the results showed that four of the five transgenic lines showed a colour band at approximately $20 \mathrm{kDa}$ corresponding to the molecular weight of the recombinant GmDREB2 protein (Fig.3C). Thus, the recombinant GmDREB2 protein was expressed in four $T_{1}$ transgenic soybean lines (T1-1, T1-6, T1-7, and T1-8) and the transformation frequency was $0.755 \%$.

All four transgenic lines (T1-1, T1-6, T1-7, and T1-8) continued to be monitored for the purpose of analysing and evaluating the functional expression of the GmDREB2 transgene at the $T_{2}$ generation.

\section{Evaluation of some morphological and biochemical characteristics of the transgenic soybean lines in the $T_{2}$ generation}

The seeds of the transgenic lines in the $T_{1}$ generation, $T 1-1$, T1-6, T1-7 and T1-8, germinated and grew into the $T_{2}$ plants T2-1, T2-6, T2-7 and T2-8. The analytical results of some morphological and biochemical characteristics such as plant height, number of pods/a tree, 100-seed weight, protein content, lipid content showed that no significant difference between the transgenic soybean lines in the $T_{2}$ generation and non-transgenic soybeans, DT84 cultivar $(\mathrm{P}<0.05)$ (Table 1). However, transgenic lines had lower tree height from 0.8 to $2.2(\mathrm{~cm})$, and two transgenic lines T2-1, T2-7 had higher lipid content from 1.17-1.21 (\%). Thus, at the $T_{2}$ generation, all four transgenic soybean lines, denoted by T2-1, T2-6, T27 , and T2-8, had normal growth and development and no difference of some morphological and biochemical characteristics compared to the DT84 original cultivar (nontransgenic plants). 
Table1. The morphological and biochemical characteristics of the transgenic soybean lines and original cultivar DT84 (nontransgenic plants).

\begin{tabular}{llllll}
\hline & DT84 & T2-1 & T2-6 & T2-7 \\
\hline Plant height $(\mathrm{cm}) ; \mathrm{n}=15$ & $30.00^{\mathrm{b}} \pm 0.45$ & $28.67^{\mathrm{ab}} \pm 0.76$ & $29.20^{\mathrm{ab}} \pm 0.55$ & $27.80^{\mathrm{a}} \pm 0.67$ & $28.07^{\mathrm{ab}} \pm 0.69$ \\
Number of pods/a plant; $\mathrm{n}=15$ & $26.00^{\mathrm{a}} \pm 0.86$ & $24.20^{\mathrm{a}} \pm 0.53$ & $25.07^{\mathrm{a}} \pm 0.77$ & $26.13^{\mathrm{a}} \pm 0.97$ & $26.73^{\mathrm{a}} \pm 0.86$ \\
100-seed weight $(\mathrm{g}) ; \mathrm{n}=3$ & $14.63^{\mathrm{ab}} \pm 0.24$ & $14.57^{\mathrm{ab}} \pm 0.18$ & $15.10^{\mathrm{ab}} \pm 0.40$ & $15.43^{\mathrm{b}} \pm 0.39$ & $14.43^{\mathrm{a}} \pm 0.12$ \\
Protein content $(\%) ; \mathrm{n}=3$ & $36.57^{\mathrm{a}} \pm 0.23$ & $35.82^{\mathrm{a}} \pm 0.15$ & $35.77^{\mathrm{a}} \pm 0.35$ & $36.26^{\mathrm{a}} \pm 0.26$ & $36.16^{\mathrm{a}} \pm 0.34$ \\
Lipid content $(\%) ; \mathrm{n}=3$ & $20.03^{\mathrm{a}} \pm 0.18$ & $21.51^{\mathrm{b}} \pm 0.15$ & $20.32^{\mathrm{a}} \pm 0.09$ & $21.47^{\mathrm{b}} \pm 0.32$ & $20.57^{\mathrm{a}} \pm 0.27$ \\
\hline
\end{tabular}

Note: The symbol \pm represents standard error.

The letters accompanying the same showed no difference by Duncan tests at $\mathrm{P}<0.05$.

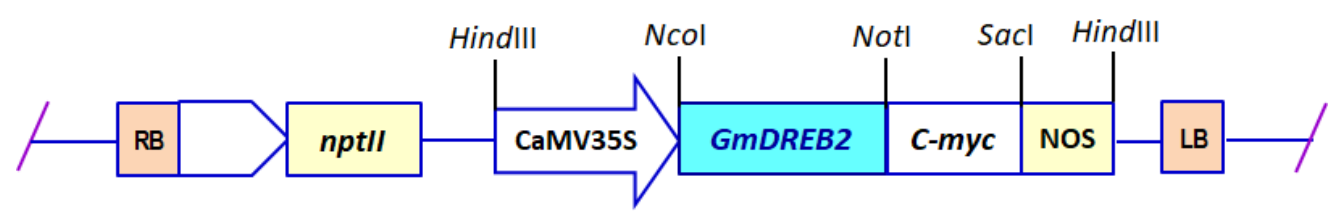

Fig 1. Diagram of the 35S- GmDREB2-cmyc construct in thepBI121- GmDREB2 vector used for Agrobacterium-mediated transformation. LB: left T-DNA border; RB: right T-DNA border; nptll:neomycin-phospo-transferase II; 35S: cauliflower mosaic virus $35 \mathrm{~S}$ promoter; GmDREB2gene isolated from mRNA of the Vietnamese soybean cultivar DT2008 (GenBank: LK936507.1);c-myc: nucleotide sequence encoding cmyc peptide; NOS: NOS- ter (terminator nopaline synthase).

Table 2. Proline content of the $T_{2}$ transgenic soybean lines and non-transgenic plants after five and nine days in drought-stress conditions.

\begin{tabular}{|c|c|c|c|c|c|c|c|c|}
\hline \multirow[t]{2}{*}{$\begin{array}{l}\text { WT and } \\
\text { soybean lines }\end{array}$} & \multirow[t]{2}{*}{ transgenic } & \multirow[t]{2}{*}{$\begin{array}{l}\text { Proline content in well-watered } \\
\text { condition }\left(\mu \mathrm{mol} . \mathrm{g}^{-1}\right)\end{array}$} & \multicolumn{3}{|c|}{$\begin{array}{l}\text { The change in proline content of transgenic soybean lines after } 5 \text { days } \\
\text { under drought-stress condition }\end{array}$} & \multicolumn{3}{|c|}{$\begin{array}{c}\text { The change in proline content of transgenic soybean lines after } 9 \\
\text { days under drought-stress condition }\end{array}$} \\
\hline & & & $\begin{array}{c}\text { PCADS } \\
\left(\mu \mathrm{mol} . \mathrm{g}^{-1}\right) \\
\end{array}$ & RICWW (\%) & RICWT (\%) & $\begin{array}{c}\text { PCADS } \\
\left(\mu \mathrm{mol}^{-1} \mathrm{~g}^{-1}\right) \\
\end{array}$ & RICWW (\%) & RICWT (\%) \\
\hline DT84" & & $1.62^{\mathrm{a}} \pm 0.04$ & $2.43^{\mathrm{a}} \pm 0.08$ & 150.00 & 100.00 & $2.83^{\mathrm{a}} \pm 0.11$ & 174.69 & 100 \\
\hline T2-1 & & $1.76^{\mathrm{a}} \pm 0.06$ & $4.16^{b} \pm 0.11$ & 186.93 & 135.39 & $3.97^{b} \pm 0.06$ & 225.57 & 140.28 \\
\hline T2-6 & & $2.67^{b} \pm 0.05$ & $4.83^{c} \pm 0.18$ & 155.81 & 171.19 & $4.82^{c} \pm 0.08$ & 180.52 & 170.32 \\
\hline $\mathrm{T} 2-7$ & & $3.26^{c} \pm 0.05$ & $6.11^{d} \pm 0.08$ & 187.42 & 251.44 & $7.62^{d} \pm 0.04$ & 233.74 & 269.26 \\
\hline
\end{tabular}

Note: "DT84 is original cultivar DT84- non-transgenic plants. The letters accompanying the same showed no difference with $\mathrm{P}<0.05$ ( $\mathrm{n}=3$ ). The symbol \pm represents standard error.

Abbreviations: PCADS_proline content after of drought-stress treatments; RICWW_rate of increase compared to well-watered condition; RICWT_rate of increase compared to non-transgenic plants; PCADS_proline content after of drought-stress treatments.

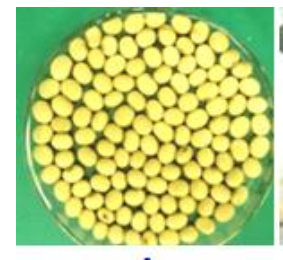

A

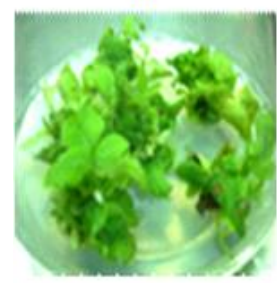

E

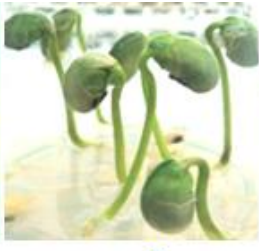

B

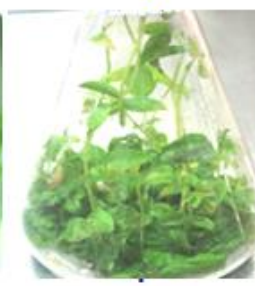

$\mathbf{F}$

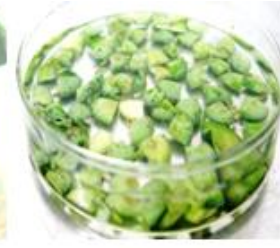

C

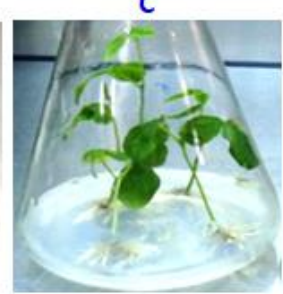

G

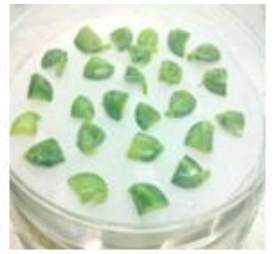

D

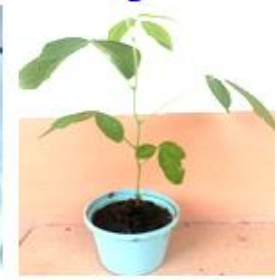

H

Fig 2. Transformation and in vitro regeneration of transgenic soybean plants from the cotyledons of soybean cultivar DT84 by infecting A. tumefaciens through the cotyledonary node. A: Soybean seeds DT84 after sterilizing; B: Seeds germinated on the GM media; C: The cotyledons were infected by A. tumefaciens carrying the 35S- GmDREB2-cmyc construct for 30 mins; D: Co- cultivated in co-cultivation media (CCM) in the dark for 5 days; $E$ : The cotyledons were cultured on SIM multi shoot regeneration media for 2 weeks (supplemented with 2 mg. $\mathrm{L}^{-1}$ BAP and $50 \mathrm{mg} \cdot \mathrm{L}^{-1}$ kanamycin); F: Cotyledons were removed and cultured on the SEM media for 2 weeks (supplemented with $0.5 \mathrm{mg} \cdot \mathrm{L}^{-1} \mathrm{GA}_{3}, 0.1 \mathrm{mg} \cdot \mathrm{L}^{-1} \mathrm{IAA}, 50 \mathrm{mg} \cdot \mathrm{L}^{-1}$ kanamycin); G: Initiation of the roots from regenerated shoots in RM media for 20 days (supplemented with $0.1 \mathrm{mg} \cdot \mathrm{L}^{-1} \mathrm{IBA}$ ); $\mathrm{H}$ : Rooted plantlets were transferred to pots containing 1:1 mixture of rice husk char and sand. 
Table 3. The nucleotide sequences of the primer pairs used for the PCR and DNA probes.

\begin{tabular}{lll}
\hline Primers & Nucleotide sequence $\left(5^{\prime}-3^{\prime}\right)$ & Size (bp) \\
\hline GmDREB2-BamHI-F/GmDREB2-cmyc- & CGGATCCGATGGAGAAGCGGGTTAGG & 526 \\
\cline { 2 - 3 } Sacl- & CGAGCTCGTCAATTCAGATCCTCTTCTG & \multirow{2}{*}{165} \\
qGmP5CS-F/qGmP5CS- $R$ & CGAACTGAGCTTGCAGAGGGGC & 152 \\
qAct-F/qAct- $R$ & TCGCTTAGCCTCCTTGCCTCC & 152 \\
\hline
\end{tabular}

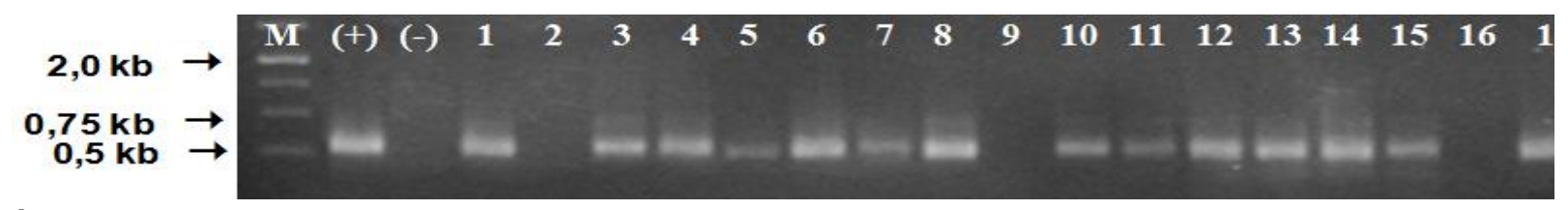

A
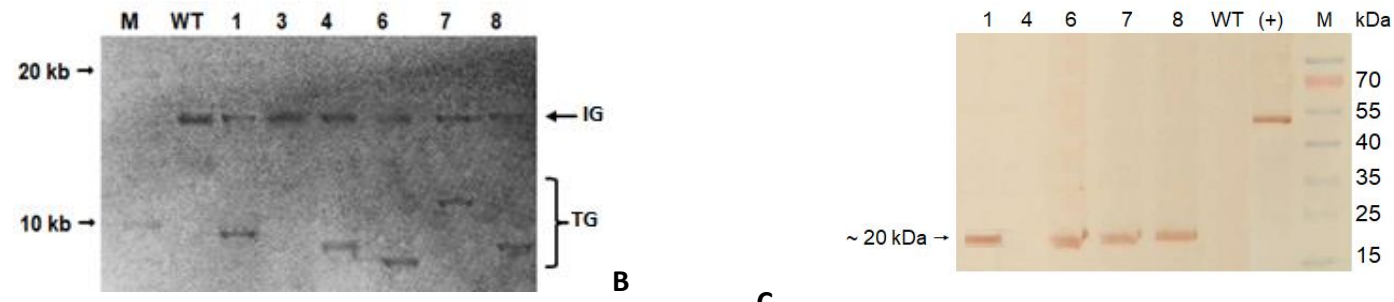

Fig 3. A: Electrophoresis results of the $P C R$ products to confirm the presence of the GmDREB2 transgene in the transgenic soybean plants and non- transgenic soybean plants. M: DNA marker $1 \mathrm{~kb} ;(+)$ : the PCR products from the plasmid $p B / 121-$ GIDREB2; (-): the PCR products from the genomic DNA of the non-transgenic soybean plants; 1- 17: the PCR products from the GmDREB2transgenic plants in the TO generation; B: Results of the Southern blot analysis to detect the presence of the GMDREB2 transgene in the genomes of the transgenic soybean plants with the biotin-labelled probe at the TO generation. M: DNA marker; WT: non-transgenic soybean plants; $1,3,4,6,7$, and 8: T0 generation transgenic soybean plants with the positive PCR product growing and developing normally, namely, T0-1, T0-3, T0-4, T0-6, T0-7 and T0-8, respectively. IG: The DNA segments contain the intrinsic GmDREB2 gene; TG: The DNA segments contain the GmDREB2 transgene. C: Western blot analysis of the recombinant GmDREB2 protein from transgenic soybean plants at the T1 generation. M: Standard protein ladder (10 - $250 \mathrm{kDa}) ; 1$, 4, 6, 7 and 8 indicate T1-1, T1-4, T1-6, T1-7, and T1-8, respectively. Recombinant GmDREB2 protein of transgenic soybean lines; (+): DAT protein $\sim 20 \mathrm{kDa}$ with the c-myc tag; WT: Protein of the non-transgenic plants.

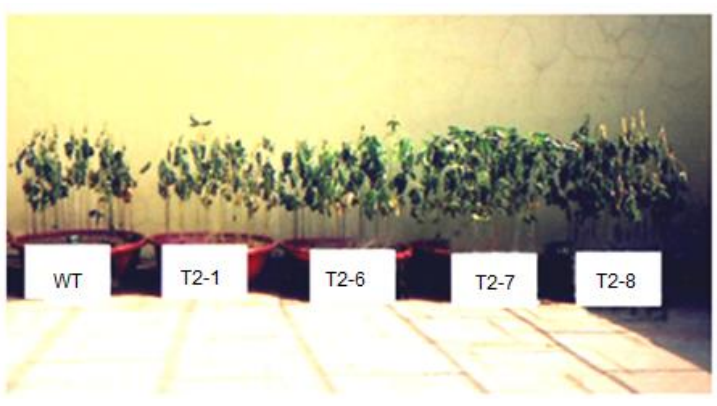

A

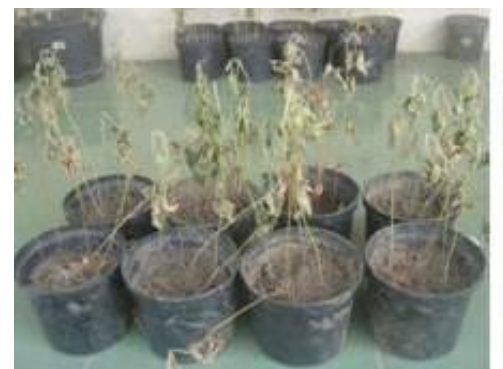

B

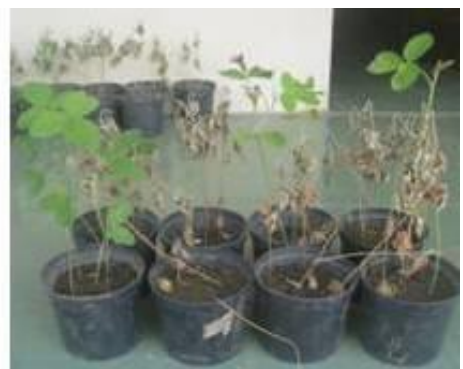

C

Fig 4. A: Transgenic soybean lines at the $T 2$ generation and non-transgenic plants after seven days in drought-stress conditions. B: The non-transgenic plants and the transgenic soybean lines after fifteen days in drought-stress conditions. C: The T2-7 transgenic soybean line at fifteen days after suspension of irrigation, and then 3 days of watering to recover. 


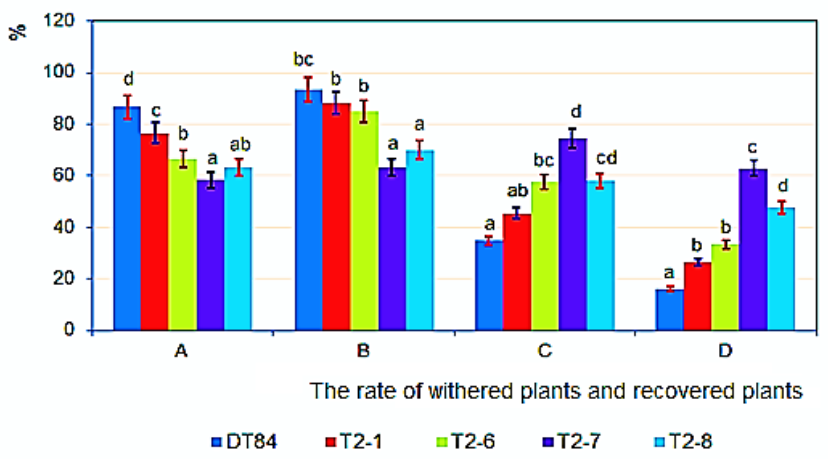

Fig 5. Responses of the transgenic lines (T2-1, T2-6, T2-7, T2-8) and DT84 cultivar (non-transgenic plants) to drought-stress. A: The rate of withered plants at 5 days after of drought-stress treatments (\%); B: The rate of plants withered at 9 days after of droughtstress treatments (\%); C: The rate of recovered plants at 5 days after of drought-stress treatments (\% of recovered plants in total the withered plants); D: The rate of recovered plants at 9 days after of drought-stress treatments (\% of recovered plants in total the withered plants). Different letters indicate significant difference by Duncan tests at $P<0.05 ;(n=20)$. Vertical bars represent standard error.

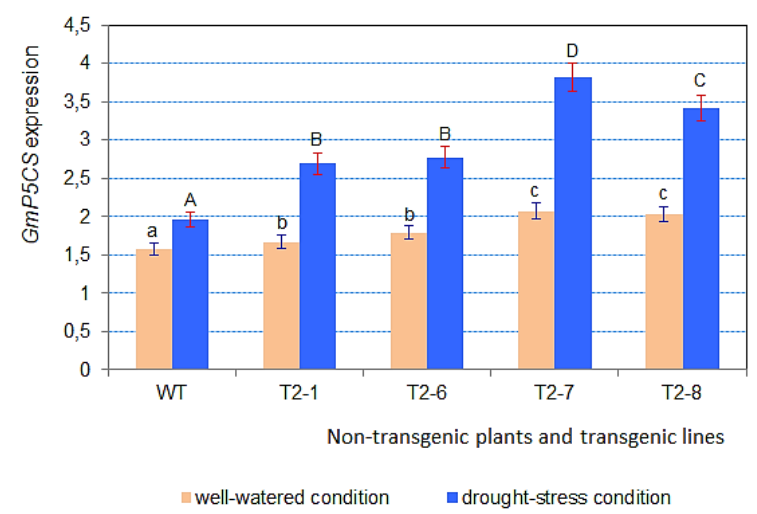

Fig 6. Expression patterns of drought stress-response GMP5CS gene in four lines of the $\mathrm{T}_{2}$ transgenic soybean and WT determined by the qRT-PCR. Actin (152 bp) was used as reference gene. GmP5CS gene (165 bp) showed increased expression levels in the transgenic soybeans compared to the WT. WT: non-transgenic plants; T2-1, T2-6, T2-7, T2-8: the transgenic soybean lines in T2 generation. Different letters above columns indicate statistically significant $(P<0.05)$ differences by Duncan's tests. The data above columns are the mean of three biological replicates \pm standard error.

\section{Evaluation of the drought tolerance of the transgenic soybean lines}

At well-watered conditions, the transgenic soybean lines in the $T_{2}$ generation showed no difference in morphology between the transgenic and non-transgenic plants; however, disparities were seen in the drought-stress conditions (Fig.4A). After 15 days under stress conditions, all plants of the four transgenic lines and non-transgenic plants had withered or died. However, after 3 days of watering to recover, there were some transgenic plants of the T2-7 line that survived (Fig.4B, 4C).

Analysis results of responses of four transgenic lines (T2-1, T2-6, T2-7, T2-8) at 5 days after of drought-stress treatments showed that four transgenic lines T2-1, T2-6, T2-7, T2-8 had the rate of plants withered less than the non-transgenic plants; but at 9 days after of drought-stress treatments, only two lines T2-7 and T2-8 had the rate of plants withered less than the non-transgenic plants $(P<0.05)$ (Fig.5). Analysis of recoverable ability of the transgenic lines, results in figure 5 showed that when plants were re-irrigated, the rate of plants recovered in four transgenic lines T2-1, T2-6, T2-7, T28 were higher than non-transgenic plants and highest was T2-7 line $(P<0.05)$.
To elucidate the putative molecular mechanisms of GMDREB2 in the drought-stress response, the expression of a drought stress-response GMP5CS gene was investigated in the transgenic lines, T2-1, T2-6, T2-7, T2-8, and nontransgenic plants under well-watered condition and drought-stress conditions, respectively. The qRT-PCR analysis results showed that GMP5CS gene was shown to have significantly $(P<0.05)$ increased expression in the transgenic soybean plants under normal and drought-stress conditions (Fig.6). The level of GmP5CS expression in the $\mathrm{T}_{2}$ transgenic lines higher than compared to non-transgenic plants with considering both in well-watered condition and stressed conditions were from 1.06 to 1.31 -fold and 1.37 to 1.95-fold, respectively.

The proline content analysis results in Table 2 showed that in the $T_{2}$ generation, the proline content of four transgenic soybean lines (T2-1, T2-6, T2-7, and T2-8) after five days in the drought-stress condition increased from $155.81 \%$ to $187.42 \%$ compared to well-watered condition, which was a greater increase than that of the non-transgenic plants from $5.81 \%$ to $37.42 \%$. Additionally, after five days under drought-stress conditions, the proline content of transgenic soybean lines T2-1, T2-6, T2-7, and T2-8 increased compared to non-transgenic plants from $135.39 \%$ to $251.44 \%$. At nine 
days after in the drought-stress condition, the proline content of four transgenic soybean lines (T2-1, T2-6, T2-7, and T2-8) increased from $180.52 \%$ to $233.74 \%$ compared to well-watered condition, which was a greater increase than that of the non-transgenic plants from $5.83 \%$ to $59.05 \%$. Additionally, after nine days in drought-stress conditions, the proline content of transgenic soybean lines T2-1, T2-6, T2-7, and T2-8 increased compared to non-transgenic plants from $140.28 \%$ to $269.26 \%$.

\section{Discussion}

The transformation technique was used to study on relationships between GmDREB2 with the transcription level of $G M P 5 C S$, the proline accumulation and abiotic-stress tolerance in soybean plants. But we noticed that high content of protein and lipid in soybean seeds can cause difficulties in in vitro culture. Therefore, selecting appropriate transformation materials for gene transfer by Agrobacterium was studied. In 1988, Maud et al. regenerated multiple buds from soybean cotyledons and created transgenic plants by using an Agrobacteriummediated gene transfer system (Maud et al., 1988), followed by Xue et al. (2006), Paz et al. (2006) and Olhoft et al. (2006, 2007). Olhoft et al. (2006) proposed the soybean transformation method via mature cotyledonary node and then Yamada et al. (2012) summarized the effectiveness of this method. The authors agreed that the shoot formation was induced successfully on soybean cotyledons in case of the Agrobacterium tumefaciens -mediated transformation. This method becomes very popular and widely applied (Kim et al., 2016; Hada et al., 2018; Park et al., 2019). However, biological scientists still improve it in order to increase transformation efficiency in soybean plants. In our study, soybean half-cotyledons were used as transforming materials.

Proline is an important amino acid adjusting the osmotic pressure. There are some genes involving the proline biosynthesis in plants. In which, P5CS is a key enzyme in its pathways (Hu et al., 1992). The expression enhancement of P5CS gene resulted in increasing proline content in the transgenic plants. Therefore, improving drought tolerance, salt tolerance of crop plants according to the approach of increase the transcription level of P5CS gene is interested in research (Soleimani et al., 2015; Guan et al., 2018). Currently, many genes in plants induced by drought, salt and cold stress. They are activated by transcription factors, such as genes encoding DREB, MYB transcription factors (Kudo et al., 2017; Wang et al., 2017). The DREB2 protein is a transcription factor that activates the transcription of drought-resistant genes, including the P5CS gene. Reports of several studies have shown that overexpression of DREB2 has improved drought tolerant ability in tobacco and wheat (Dao et al., 2015; Eftekhari et al., 2017).

In this study expression of GMP5CS gene was investigated in transgenic soybean plants with GmDREB2 transgene in both well-watered condition and drought-stress conditions. The level of GMP5CS expression in the $\mathrm{T}_{2}$ transgenic lines was higher than those compared to non-transgenic plants. Results of enhanced expression of GMP5CS gene that increased stress tolerance of transgenic potato, wheat as a result of the increased proline content have been reported in some previous studies (Hmida-Sayari et al., 2005; Vendruscolo et al., 2007).

Analysis of free proline content in transgenic tobacco plants indicated that the overexpression of DREB2 accumulated higher levels of free proline compared to the wild type plants under drought conditions (Chen et al., 2007; Dao et al., 2015). In our study, the proline content of young soybean plants in transgenic lines and non-transgenic plants increased in drought stress conditions compared to wellwatered condition. The proline content of transgenic lines had a higher increase in rate than non-transgenic plants, increasing from 1.35 to 2.51 -fold (after five days in drought stress condition) and from 1.40 to 2.69 -fold (after nine days under drought stress condition) compared to non-transgenic plants. Thus, overexpression of GMDREB2 led to the upregulated expression of drought-stress responsive GmP5CS gene, increased accumulation of free proline, and enhanced tolerance to drought-stress in transgenic soybean plants. This result could suggest that the expression of genes involved in proline biosynthesis and adjusted osmotic pressure in the cell had been activated by the DREB2 transcription factor. However, DREB2 might be involved in many pathways, in addition to combination with DRE region, including regulation of miRNAs and other pathways (Zhang et al., 2013). Therefore, it is necessary to continue researching to clarify the role of DREB2 transcription factor for drought tolerance of soybean plants. Our research results indicated that GmDREB2 gene overexpression may be useful in improving plant tolerance to drought stresses and can contribute to the prospect of producing the drought tolerant soybean cultivars, especially considering the conditions of global climate change and the EI Niño phenomenon.

\section{Materials and methods}

\section{Plant materials and transgenic construct}

The cotyledons of the DT84 Vietnamese soybean cultivar was used for Agrobacterium-mediated transformation with 35S- GmDREB2- cmyc construct (Fig. 1). Sterilized soybean seeds were used for in vitro germination and collecting cotyledon. The cotyledons were separated and the petiole was removed to obtain the half-seed explant. Cotyledonary node was wounded by using blade, then infected with recombinant $A$. tumefaciens. The GmDREB2 gene in the $35 S$ GmDREB2- cmyc construct isolated from the DT2008 cultivar with high drought tolerance (Ha et al., 2013; Sulieman et al., 2015). The code of GmDREB2 gene on GenBank is LK936507.1 (Dao et al., 2014).

\section{Agrobacterium-mediated transformation}

The A. tumefaciens-mediated transformation of the 35SGmDREB2-cmyc construct into the soybeans via cotyledonary node infection, regeneration and selection of the transgenic soybean plants were performed as described by Olhoft et al. (Olhoft et al., 2006, 2007). The recombinant A. tumefaciens CV58 strain containing the pBI121-GMDREB2 vector was cultured in LB media and collected by centrifugation with an $\mathrm{OD}_{660 \mathrm{~nm}}=0.8$. After being wounded, cotyledons were immersed in an A. tumefaciens suspension for $40 \mathrm{~min}$ and co-cultivated in co-cultivation media (CCM 
media: $0.316 \mathrm{~g} . \mathrm{L}^{-1} \mathrm{~B} 5$ salt, $3.9 \mathrm{~g} . \mathrm{L}^{-1} \mathrm{MES}, 30 \mathrm{~g} . \mathrm{L}^{-1}$ sucrose, $5 \mathrm{~g} . \mathrm{L}^{-1}$ agar, $1.0 \mathrm{mg} . \mathrm{L}^{-1} \mathrm{~B} 5$ vitamin, $0.2 \mathrm{mM} \mathrm{AS}, 400 \mathrm{mg} \cdot \mathrm{L}^{-1} \mathrm{~L}-$ cysteine, $158 \mathrm{mg} \cdot \mathrm{L}^{-1}$ sodium thiosulfate, $154 \mathrm{mg} \cdot \mathrm{L}^{-1} \mathrm{DTT}, 0.25$ mg. $\mathrm{L}^{-1} \mathrm{GA} 3$, and $1.5 \mathrm{mg} \cdot \mathrm{L}^{-1} \mathrm{BAP} ; \mathrm{pH}=5,4$ ) in the dark for 5 days. The cotyledons were cultured on SIM multi-shoot regeneration media (0.3052 g.L ${ }^{-1}$ B5 salt, 0.59 g.L ${ }^{-1}$ MES, 30 g. $\mathrm{L}^{-1}$ sucrose, $1.0 \mathrm{mg} . \mathrm{L}^{-1} \mathrm{~B} 5$ vitamin, $2 \mathrm{mg} . \mathrm{L}^{-1} \mathrm{BAP}$, and 50 mg. $L^{-1}$ kanamycin) for 2 weeks. Cotyledons were removed and cultured on SEM media (4.3 g. $\mathrm{L}^{-1} \mathrm{MS}, 0.59 \mathrm{~g} . \mathrm{L}^{-1} \mathrm{MES}, 30$ g. $\mathrm{L}^{-1}$ sucrose, 5 g. $\mathrm{L}^{-1}$ agar, $1.0 \mathrm{mg} . \mathrm{L}^{-1}$ B5 vitamin, $50 \mathrm{mg} \cdot \mathrm{L}^{-1} \mathrm{~L}$ asparagine, $100 \mathrm{mg} \cdot \mathrm{L}^{-1} \mathrm{~L}$-pyron glutamic acid, $0.5 \mathrm{mg} \cdot \mathrm{L}^{-1} \mathrm{GA} 3$, $0,1 \mathrm{mg} \cdot \mathrm{L}^{-1} \mathrm{IAA}$, and $50 \mathrm{mg} \cdot \mathrm{L}^{-1}$ kanamycin) for 2 weeks. The shoots $(2-3 \mathrm{~cm})$ were cultured on RM media (1.58 g. $\mathrm{L}^{-1} \mathrm{MS}$, $0.59 \mathrm{~g} . \mathrm{L}^{-1} \mathrm{MES}, 20 \mathrm{~g} . \mathrm{L}^{-1}$ sucrose, $5.0 \mathrm{~g} . \mathrm{L}^{-1}$ agar, $0.1 \mathrm{mg} . \mathrm{L}^{-1} \mathrm{IBA}$, and $1.0 \mathrm{mg} . \mathrm{L}^{-1} \mathrm{~B} 5$ vitamin) for 20 days to form roots.

The plants that were regenerated from the transformed samples (cotyledons) in vitro were designated as the $T_{0}$ transgenic generation. The seeds of transgenic plants in the $\mathrm{T}_{0}$ generation germinated and grew into plants; these were considered the $T_{1}$ transgenic generation. The seeds of transgenic plants in the $T_{1}$ generation germinated and grew into plants; these were considered the $T_{2}$ transgenic generation.

\section{Analysis of transgenic plants using PCR, Southern blotting} and Western blotting

Total DNA was extracted from young leaves based on the method used by Saghai-Maroofet et al. (1984). PCR analysis was used to confirm the presence of the GMDREB2 transgene in the transgenic soybean plants in the $T_{0}$ generation, with the primer pair GmDREB2-BamHI/ GmDREB2-cmyc-Sacl (Table 3). The transgenic soybean plants which were PCR-positive were subjected to Southern blot analysis (Southern, 1975). The transgenic soybean plants in the $T_{0}$ generation with the positive Southern blot were analysed for expression of the recombinant GmDREB2 protein in the $T_{1}$ generation by $10 \%$ SDS-PAGE (Laemmli, 1970) and Western blot (Sun et al., 2006).

\section{Analysis of some characteristics of transgenic and non- transgenic soybeans}

Transgenic soybean and non-transgenic soybean seedlings cultivated into $3.0 \mathrm{~kg}$ pots containing soil: manure: sand (2: 2: 1 ) and at well- watered conditions. The characteristics of transgenic soybeans with non-transgenic soybeans being analyzed such as plant height, number of pods, 100-seed weight, protein content and lipid content of seeds.

\section{Drought-stress treatment and evaluation of drought-stress tolerance of transgenic plants}

Drought-stress tolerance of transgenic plants and nontransgenic soybeans being analyzed based on the rate of withered trees at drought-stress, the rate of surviving plants at re-irrigated, and proline content analysis at two water regimes, well-watered and drought-stress conditions.

Analysis of some morphological characteristics of transgenic soybeans and non-transgenic soybeans conducted at drought stress conditions. The seeds of the transgenic lines in the $T_{1}$ generation germinated and grew into the $T_{2}$ plants in the clean sand conditions. When soybean plants have 3 leaves then initiation drought stressed treatments. Prior to the drought-stress initiation, the pots were saturated and thereafter irrigation was stopped for five and nine days, and the number of withered trees was counted and calculated as a percentage. Plants were re-irrigated and 3 days after the number of surviving plants was counted and calculated the percentage of trees recovered in total the trees withered.

\section{Analysis for transcriptional expression of GMP5CS gene and determine proline content in transgenic plants}

The qRT-PCR was used for transcriptional expression analysis of GMP5CS gene in the non-transgenic soybeans and the GmDREB2 transgenic soybean lines at two water regimes, well watered and drought-stress conditions. The actin is reference gene. Calculate the expression level (R) of GMP5CS gene by using the $R=2^{-\Delta \Delta C t}$ method according to Livak and Chmittgen (2001).

The proline content of transgenic plants and non-transgenic plants after five and nine days under drought-stress conditions were determined by the method of Bateset el al. (1973). Proline content was expressed as $\mu \mathrm{mol} . \mathrm{g}^{-1}$ of fresh mass.

\section{Statistical analysis}

The data obtained from measurements of selected the morphological and biochemical characteristics such as the plant height, number of pods, 100-seed weight, protein content and lipid content of seeds, the rate of withered trees at drought-stress, the rate of surviving plants at reirrigated, proline content and transcriptional expression were statistically processed and the significant difference between the transgenic lines and non-transgenic plants were tested by Duncan's tests at the $P<0.05$.

\section{Conclusions}

The 35S-GmDREB2-cmyc construct was successfully transformed and produced transgenic soybean lines and incorporation of the GMDREB2 transgene into the soybean genome has been demonstrated. The recombinant GmDREB2 protein of approximately $20 \mathrm{kDa}$ was expressed in four $\mathrm{T}_{1}$ transgenic soybean lines, and transgenic performance was $0.755 \%$. In the $T_{2}$ generation, the transgenic soybean lines, T2-1, T2-6, T2-7, and T2-8, had normal growth and development and no difference compared to the non-transgenic plants. The proline content of four transgenic soybean lines in the $T_{2}$ generation, $T 2-1$, $\mathrm{T} 2-6, \mathrm{~T} 2-7$, and T2-8 after being five, nine days in droughtstress conditions, respectively, which was increased from 1.35 to 2.51 - fold and $1.40-2.69$-fold, compared to that in non-transgenic plants. The transgenic lines T2-1, T2-6, T2-7, and T2-8 had higher drought-resistance than non-transgenic plants; and two transgenic soybean lines T2-7 and T2-8 lines had the greatest drought tolerance.

\section{Acknowledgements}

This research is funded by Vietnam National Foundation for Science and Technology Development (NAFOSTED) under grant number 106.01-2018.27. The authors would like to express their gratefulness for the help the Key Laboratory of 
Gene Technology, Institute of Biotechnology, Vietnam Academy of Science and Technology.

\section{References}

Bates LS, Waldren RP, Teare ID (1973) Rapid determination of free proline for water-stress studies. Plant Soil. 39:205207.

Bray EA (2004) Genes commonly regulated by water-deficit stress in Arabidopsis thaliana. Exp Bot. 55:2331-2341.

Chen M, Wang QY, Cheng XG, Xu ZS, Li LC, Ye XG, Xia LQ, Ma YZ (2007) GmDREB2 a soybean DRE-binding transcription factor, conferred drought and high-salt tolerance in transgenic plants. Biochem Biophys Res Commun. 353:299-305.

Chu HM, Nguyen TTH, Chu HL, Nguyen TA, Le VS, Chu HH (2010) Characteristics of the gene encoding pyrroline-5carboxylate synthase (P5CS) in Vietnamese soybean cultivars (Glycine max L. Merrill). International Conference on Biology, Environment and Chemistry 2010:319-323.

Dao TX, Ho MT, Vu TTT, Le VS, Chu HM (2015) Cloning and Overexpression of GmDREB2 Gene from a Vietnamese Drought-resistant Soybean Variety. Braz Arch Biol Technol.58:651-657.

Dao TX, Hoang HTT, Ho TM, Hoang HP, Vu TTT, Le SV, Chu $\mathrm{MH}$ (2014) Glycine max mRNA for DREB2 factor (DREB2 gene), cultivar DT2008. GenBank: LK9365071; https://www.ncbi.nlm.nih.gov/nuccore/LK936507.

Eftekhari A, Baghizadeh A, Yaghoobi MM, Abdolshahi R (2017) Differences in the drought stress response of DREB2and CAT1genes and evaluation of related physiological parameters in some bread wheat cultivars, Biotechnology \& Biotechnological Equipment, 31:4, 709716.

Guan C, Huang YH, Cui X, Liu SJ, Zhou YZ, Zhang YW (2018) Overexpression of gene encoding the key enzyme involved in proline-biosynthesis (PuP5CS) to improve salt tolerance in switch grass (Panicum virgatum L.). Plant Cell Rep.37(8):1187-1199.

Ha CV, Le DT, Nishiyama R, Watanabe Y, Tran UT, Dong NV (2013) Characterization of the newly developed soybean cultivar DT2008 in relation to the model variety W82 reveals a new genetic resource for comparative and functional genomics for improved drought tolerance. Biomed Res Int. 2013:8.

Hada A, Krishnan V, Mohamed Jaabir MS, Kumari A, Jolly M, Prveen S, Sachdev A (2018) Improved Agrobacterium tumefaciens-mediated transformation of soybean [Glycine max (L.) Merr.] following optimization of culture conditions and mechanical techniques. In Vitro Cellular \& Developmental Biology - Plant54: 672-688.

Hmida-Sayari A, Gargouri-Bouzid R, Bidani A, Jaoua L, Savoure A, Jaoua $S$ (2005) Overexpression of $\Delta 1$-pyrroline5 -carboxylate synthetase increases proline production and confers salt tolerance in transgenic potato plants. Plant Sci. 169:746-752.

Hu CAA, Delauney AJ, Verma DPS (1992) A biofunctional enzyme ( $\Delta 1$-pyrroline-5-carboxylate synthetase) catalyzes the first two steps in proline biosynthesis in plants. Proc Nat Acad Sci USA. 89:9354-9358.

Hussain SS, Ali M, Ahmad M, Siddique KH (2011) Polyamines: natural and engineered abiotic and biotic stress tolerance in plants. Biotechnol Adv. 29:300-311.
James D, Borphukan B, Fartyal D, Ram B, Singh J, Manna M, Sheri V, Panditi V, Yadav R, Achary VMM, Reddy MK. (2018) Concurrent overexpression of OsGS1;1 and OsGS2 genes in transgenic Rice (Oryza sativa L.): Impact on tolerance to abiotic stresses. Front Plant Sci. 9: 786.

Janiak A, Kwaśniewski M, Szarejko I (2016) Gene expression regulation in roots under drought. J Exp Bot. 67:1003-14.

Kim HJ, Kim MJ, Pak JH, Im HH, Lee DH, Kim KH, Lee JH, Kim DH, Choi HK, Jung HW, Chung HW (2016) RNAi-mediated Soybean mosaic virus (SMV) resistance of a Korean Soybean cultivar Plant Biotechnology Reports. 10: 257267.

Kudo M, Kidokoro S, Yoshida T, Mizoi J, Todaka D, Fernie AR, Shinozaki K, Yamaguchi-Shinozaki K (2017) Double overexpression of DREB and PIF transcription factors improves drought stress tolerance and cell elongation in transgenic plants. Plant Biotechnol J. 15: 458-471.

Kobayashi F, Ishibashi M, Takumi S (2008) Transcriptional activation of Cor/Lea genes and increase in abiotic stress tolerance through expression of a wheat DREB2 homolog in transgenic tobacco. Transgenic Res. 17:755-767.

Laemmli UK (1970) Cleavage of structural protein during the assembly of the head of bacteriophage T4. Nature 227:680-685.

Li XP, Tian AG, Luo GZ, Gong ZZ, Zhang JS, Chen SY (2005) Soybean DRE-binding transcription factors that are responsive to abiotic stresses. Theor Appl Genet. 110:1355-1362.

Livak KJ, Schmittgen TD (2001) Analysis of relative gene expression data using real-time quantitative PCR and the 2(-Delta Delta C(T)) method. Methods 25:402 - 408.

Lo Thanh Son, Le Van Son, Nguyen Vu Thanh Thanh, Chu Hoang Mau (2014) Cloning and Designing Vector Carrying GmEXP1 Gene Isolated from Local Soybean Cultivar Sonla, Vietnam. IJBBB 4: 191 - 194.

Lo TS, Le HD, Nguyen VTT, Chu HH, Le VS, Chu HM (2015) Overexpression of a soybean expansin gene, GmEXP1, improves drought tolerance in transgenic tobacco. Turk J Bot. 39: 988-995.

Maud A. W. Hinchee, Dannette V. Connor-Ward, Christine A. Newell, Raymond E. McDonnell, Shirley J. Sato, Charles S. Gasser, David A. Fischhoff, Diane B. Re, Robert T. Fraley, Robert B. Horsch (1988) Production of Transgenic Soybean Plants Using Agrobacterium-Mediated DNA Transfer. Nature Biotechnology 6: 915-922.

Mizoi J, Shinozaki K, Yamaguchi-Shinozaki K (2012) AP2/ERF family transcription factors in plant abiotic stress responses. Biochim Biophys Acta. 1819:86-96.

Olhoft PM, Donovan CM, Somers DA (2006) Soybean (Glycine max) transformation using mature cotyledonary node explants. Methods Mol Biol. 343:385-396.

Olhoft PM, Bernal LM, Grist LB, Ozias Akins P (2007) A novel Agrobacterium rhizogenes-mediated transformation method of soybean [Glycine max (L.) Merrill] using primary-node explants from seedlings. In Vitro Cell Dev Biol Plant. 43:536-549.

Park JS, Kim HJ, Cho HS, Jung HW, Cha JY, Yun DJ, Oh SW, Chung YS (2019) Overexpression of AtYUCCA6 in soybean crop results in reduced ROS production and increased drought tolerance. Plant Biotechnol Rep (2019) 13: 161168.

Paz MM, Martinez JC, Kalvig AB, Fonger TM, Wang K (2006) Improved cotyledonary node method using an alternative 
explant derived from mature seed for efficient Agrobacterium - mediated soybean transformation. Plant Cell Rep. 25: 206-213.

Saghai-Maroof MA, Soliman KM, Jorgensen RA, Allard RW (1984) Ribosomal DNA spacer-length polymorphisms in barley: Mendelian inheritance, chromosomal location and population dymnamics.Proc Natl Acad Sci USA. 81:80148018.

Soleimani V, Ahmadi J, Golkari S, Sadeghzadeh B (2015) Expression profiling of PAP3, BZIP, and P5CS genes in soybean under drought stress conditions. Turk J Bot 39: 952-961.

Southern EM (1975) Detection of specific sequences among DNA fragments separated by gel electrophoresis. J Mol Biol.98:503-517.

Sulieman S, Ha CV, Nasr Esfahani M, Watanabe Y, Nishiyama R, Pham BCT (2015) DT2008: A promising new genetic resource for improved drought tolerance in soybean when solely dependent on symbiotic $\mathrm{N}_{2}$ fixation. BioMed Res Int. ID 687213:7.

Sun HJ, Cui ML, Ma B, Ezura H (2006) Functional expression of the taste modifying protein, miraculin, in transgenic lettuce. FEBS Lett.580:620-626.

Tang MJS, Liu Y, Chen F, Shen S (2007) Isolation and functional characteri zation of the JCERF gene, a putative AP2/EREBP domain containing transcription factor, in the woodyoil plant Jatrophacurcas. Plant Mol Biol. 163:419428.
Vendruscolo EC, Schuster I, Pileggi M, Scapim CA, Molinari HB, Marur CJ, Vieira LG (2007) Stress-induced synthesis of proline confers tolerance to water deficit in transgenic wheat. J Plant Physiol. 164:1367-1376.

Zhang XX, Tang YJ, Ma QB, Yang CY, Mu YH, Suo HC, Luo LH, Nian $H$ (2013) OsDREB2A, a Rice transcription factor, significantly affects salt tolerance in transgenic soybean. PLoS ONE. 8(12): e83011:

Zhang GC, Zhu WL, Gai JY, Zhu YL, Yang LF (2015) Enhanced salt tolerance of transgenic vegetable soybeans resulting from overexpression of a novel $\triangle^{1}$-Pyrroline-5carboxylate synthetase gene from Solanum torvum Swartz. Hort. Environ. Biotechnol. 56:94-104.

Wang N, Zhang W, Qin M, Li S, Qiao M, Liu Z, Xiang F(2017) Drought Tolerance conferred in soybean (Glycine max. L) by GmMYB84, a novel R2R3-MYB transcription factor. Plant Cell Physiol. 58:1764-1776.

Xue R. G., Xie H. F., Zhang B. (2006) A multi-needle-assisted transformation of soybean cotyledonary node cells. Biotechnol. Lett., 28: 1551-1557.

Yamada T, Takagi K, Ishimoto M (2012) Recent advances in soybean transformation and their application to molecular breeding and genomic analysis. Breeding Science 61: 480494. 